\title{
CD10 and HHF35 actin in the differential diagnosis between Collagenous Spherulosis and Adenoid-Cystic Carcinoma of the breast
}

\author{
Daniela Cabibi, Antonino Giulio Giannone*, Beatrice Belmonte, Francesco Aragona, Federico Aragona \\ Department of Sciences for Health Promotion, Section of Anatomic Pathology, University of Palermo, Italy
}

\section{A R T I C L E I N F O}

\section{Article history:}

Received 9 February 2012

Received in revised form 31 March 2012

Accepted 3 May 2012

\section{Keywords:}

Breast

Collagenous Spherulosis

Adenoid-Cystic Carcinoma

CD10

HHF35

\begin{abstract}
A B S T R A C T
Collagenous Spherulosis (CS) and Adenoid-Cystic Carcinoma (AdCC) of the breast consist of cribriform proliferations of epithelial and myoepithelial cells with an immunophenotypic overlap of some myoepithelial markers, such as p63 and smooth muscle actin (SMA). To our knowledge, CD10 and HHF35 actin have not been assessed in the differential diagnosis of these two breast lesions.

We performed an immmunohistochemical study on 6 cases of CS and 9 cases of AdCC.

We found CD10, muscle-specific actin (HHF35), Estrogen and Progesterone receptors (ER and PR) to be strongly expressed in CS, but not in AdCC; C-kit was diffusely positive in AdCC and scanty in CS; SMA, p63 and Cytokeratine 5/6 (CK5/6) were positive in both.

Our results also confirm that AdCC could be true basal-like neoplasia, probably arising from a basal stem line tending to divergent differentiation toward CK5/6/C-kit+, ER/PR-, epithelial basal-like cell type, and toward a myoepitelial-like cell type, with an incomplete SMA/p63+, CD10/HHF35- immunophenotype. By contrast, CS is a reactive, benign proliferation of two well-differentiated cell types: epithelial (ER/PR+, C-kit-) and myoepithelial cells with a complete immunophenotype including CD10/HHF35 positivity.

Our study highlights the usefulness of CD10 and HHF35 in the differential diagnosis and helps to understand the histogenesis of the two lesions.
\end{abstract}

(c) 2012 Elsevier GmbH. All rights reserved.

\section{Introduction}

CS of the breast is an uncommon benign lesion described by Clement in 1987 [2], usually representing an incidental finding in breast samples removed for other lesions, including sclerosing adenosis, radial scar, intraductal papilloma, fibroadenoma, atypical ductal hyperplasia, ductal carcinoma in situ and lobular carcinoma in situ [12]. Due to its rarity and to its morphological features, it is sometimes overlooked or misdiagnosed as an atypical or malignant lesion.

CS is characterized by a cribriform growth mimicking other breast lesions, such as intraductal or invasive cribriform carcinoma and adenoid cystic carcinoma (AdCC) [8]. It consists of a mixture of epithelial and myoepithelial cells, the latter surrounding spaces often containing eosinophilic material bordered by a peripheral eosinophilic cuticle [2,7,12].

\footnotetext{
* Corresponding author at: Dipartimeno di Scienze per la Promozione della Salute, Sezione di Anatomia Patologica, A.O.U. Policlinico “P. Giaccone”, Via del Vespro 129 - 90127, Palermo, Italy. Tel.: +39 91 6553529/6553533.

E-mail address: aggianno@alice.it (A.G. Giannone).
}

Some myoepithelial markers (p63, SMA) turned out to be useful in the differential diagnosis between CS and cribriform ductal carcinoma in situ, where the myoepithelial cells are diminished and limited to a subtle row at the periphery of the ducts, and invasive carcinoma in which they are absent [6]. The differential diagnosis with AdCC still represents the most difficult challenge [11]. Both lesions consist of cribriform proliferations of epithelial and myoepithelial cells forming a sieve-like pattern of pseudocysts containing acellular basement membrane material $[2,8,11]$ and show an immunophenotypic overlap of some myopithelial markers (p63, SMA) inducing a potential diagnostic pitfall [11]. The usefulness of CD10 and HHF35 in this setting has not been assessed.

CD10 was reported to be expressed in the myoepithelial cells of the normal ducts [4], in most of the benign breast lesions and in the "in situ carcinoma", but not in invasive carcinomas [9]. CD10 expression has been recently investigated in salivary glands AdCC, where it stains negatively in $100 \%$ of cases [10]. To our knowledge, no studies are present in the literature on CD10 and HHF35 expression in breast AdCC and their usefulness in the differential diagnosis with CS.

In this study, we assessed CD10 and HHF35 expression in CS and ADCC and compared them with SMA, p63, c-kit (CD117), CK5/6, ER 
and PR, with the aim to add other potential useful markers to the differential diagnosis between the two lesions.

\section{Materials and methods}

This is a retrospective study performed on formalin-fixed, paraffin-embedded breast specimens retrieved from the Archives of the Department of Pathology, University of Palermo (Italy).

Hematoxylin-Eosin slides of 9 cases of cribriform AdCC and 6 cases of CS, diagnosed during the period 2003-2009, were reviewed among a casuistry of 4540 surgically excised breast lesions.

All the cases of AdCC were evident on mammography with associated microcalcifications.

The cases of CS were incidental findings associated with benign epithelial proliferations, such as sclerosing adenosis (2 cases), fibroadenomas ( 2 cases), and intraductal papillomas ( 1 case). Only one case showed an area of "in situ lobular neoplasia".

The mean age of the women was 56 for AdCC (range 45-67) and 55 for SC (range 42-68).

In all cases, additional sections were stained with Periodic Acid-Schiff and Alcian blue at $\mathrm{pH} 2.5$.

Immunoreactivity for CK5/6 (clone CK5/6.007, dilution $1: 100$, Biocare Medical), SMA (clone $\alpha \mathrm{sm}-1$, dilution 1:50, Novocastra), HHF35 (clone HHF35, dilution 1:400, Dako), p63 (clone 4A4, dilution 1:40, Dako), c-kit (clone T595, dilution 1:40, Novocastra), CD10 (clone 56C6, dilution 1:100, Novocastra), E-cadherin (clone 36B5, dilution 1:40, Novocastra), ER (clone 6F11, dilution 1:50, Novocastra) and PR (clone 1A6, dilution 1:40, Novocastra) was assessed on $10 \%$ formalin-fixed, paraffin-embedded sections by using the avidin-biotin complex technique according to the manufacturer's instructions (Universal LSAB kit, Dako).

In each case, normal breast myoepithelium, adjacent to the lesions, served as a positive internal control for p63, SMA, HHF35 and CD10. Stromal mast-cells served as a positive internal control for c-kit.
Normal breast epithelium served as a positive internal control for CK5/6, ER and PR receptors.

Negative controls without primary antibodies were included in each immunohistochemical run.

\section{Results}

All CS cases had been incidental findings and satisfied the criteria of Clement et al. [2].

They were circumscribed, non-encapsulated solid lesions with the maximum diameter ranging from $0.3 \mathrm{~cm}$ to $0.9 \mathrm{~cm}$.

Histological examination showed a cribriform proliferation with central spaces surrounded by thin strands of flattened cells and by mono- or multilayers of haphazardly arranged cuboidal cells in the periphery, with uniform, bland nuclei. The central spaces contain "spherules" of loosely or radial arranged eosinophilic material, positive for Alcian Blue and/or PAS (Fig. 1).

On immunohistochemical examination, the cells surrounding the spherules were positive for basal/myoepithelial markers including CK 5/6, p63, SMA, CD10 and HHF35 (Figs. 2-4).

It is noteworthy that CD10 and HHF35 were strongly positive in the inner flat layer, in the intra-cystic amorphous material and in the peripheral cuticle, but negative in the outer cuboidal cells (Fig. 4).

C-kit was negative or scanty positive only in rare ductular structures, whereas ER and PR were strongly positive (Fig. 3).

AdCC cases showed histological and histochemical features similar to CS. A cribriform pattern was present, composed of cystic spaces, containing Alcian-PAS-positive basement membrane material, surrounded by small myoepithelial-like basaloid cells and larger, cubic epithelial cells forming ductules with a random distribution (Fig. 1).

A positive immunoreactivity for SMA, p63 and CK5/6 was found (Fig. 2). C-kit pointed out several small ducts. Differently from
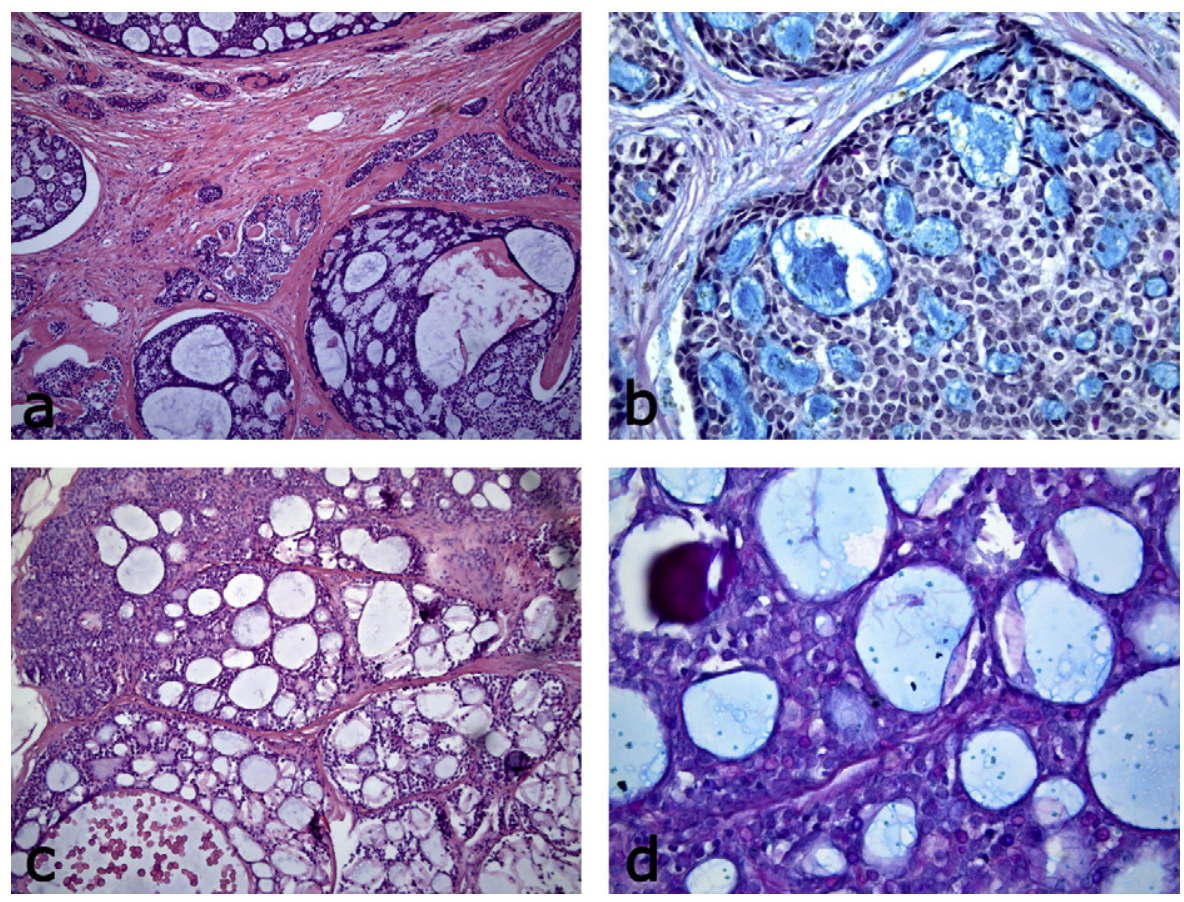

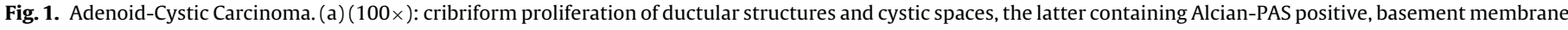

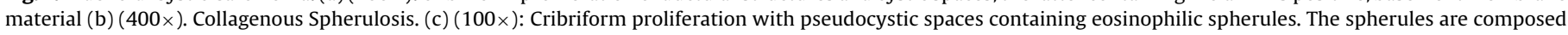
of acellular, Alcian-PAS positive material, with a darker peripheral cuticle (d) $(400 \times)$. 

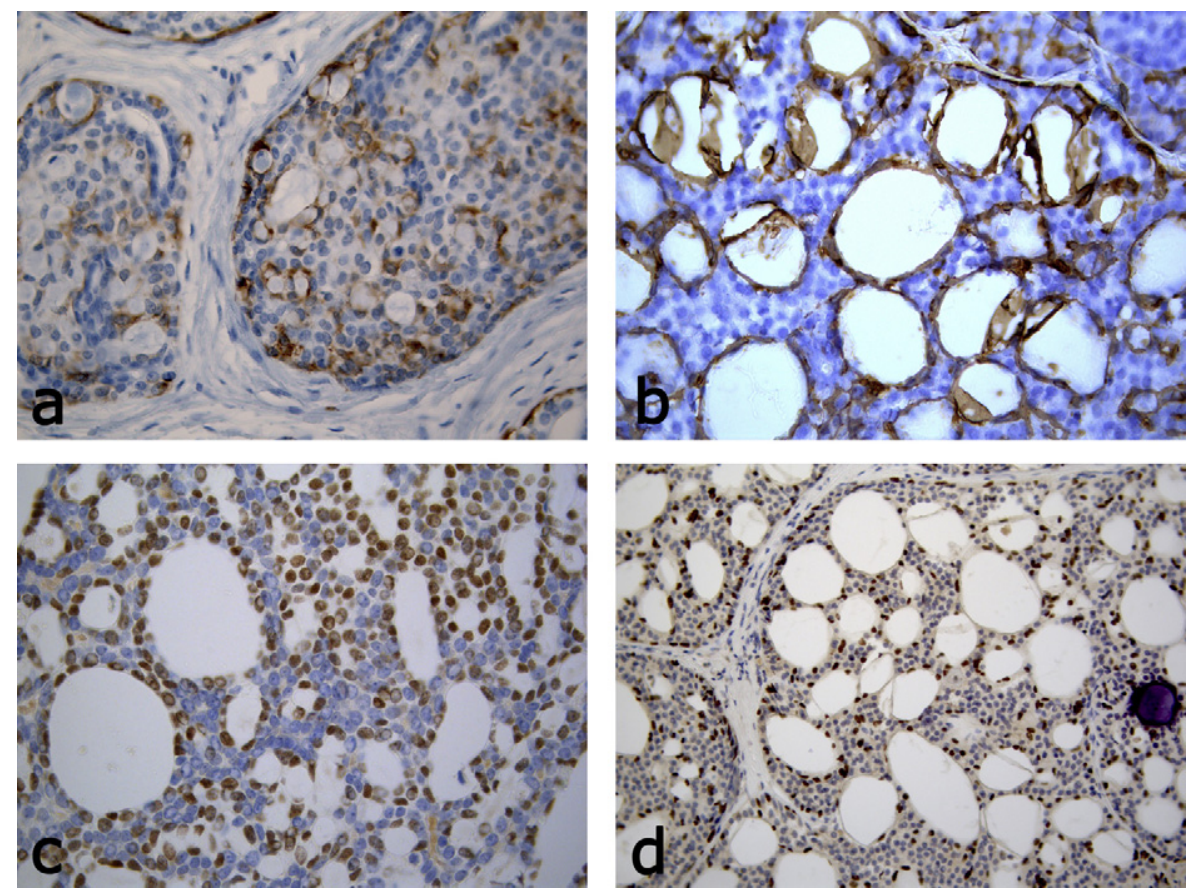

Fig. 2. Immunophenotypic overlap for SMA and p63 in myoepithelial-like cells of AdCC (a, c) (each $400 \times$ ) and in normal myoepithelial cells of CS (b, d) (400×, 200×).
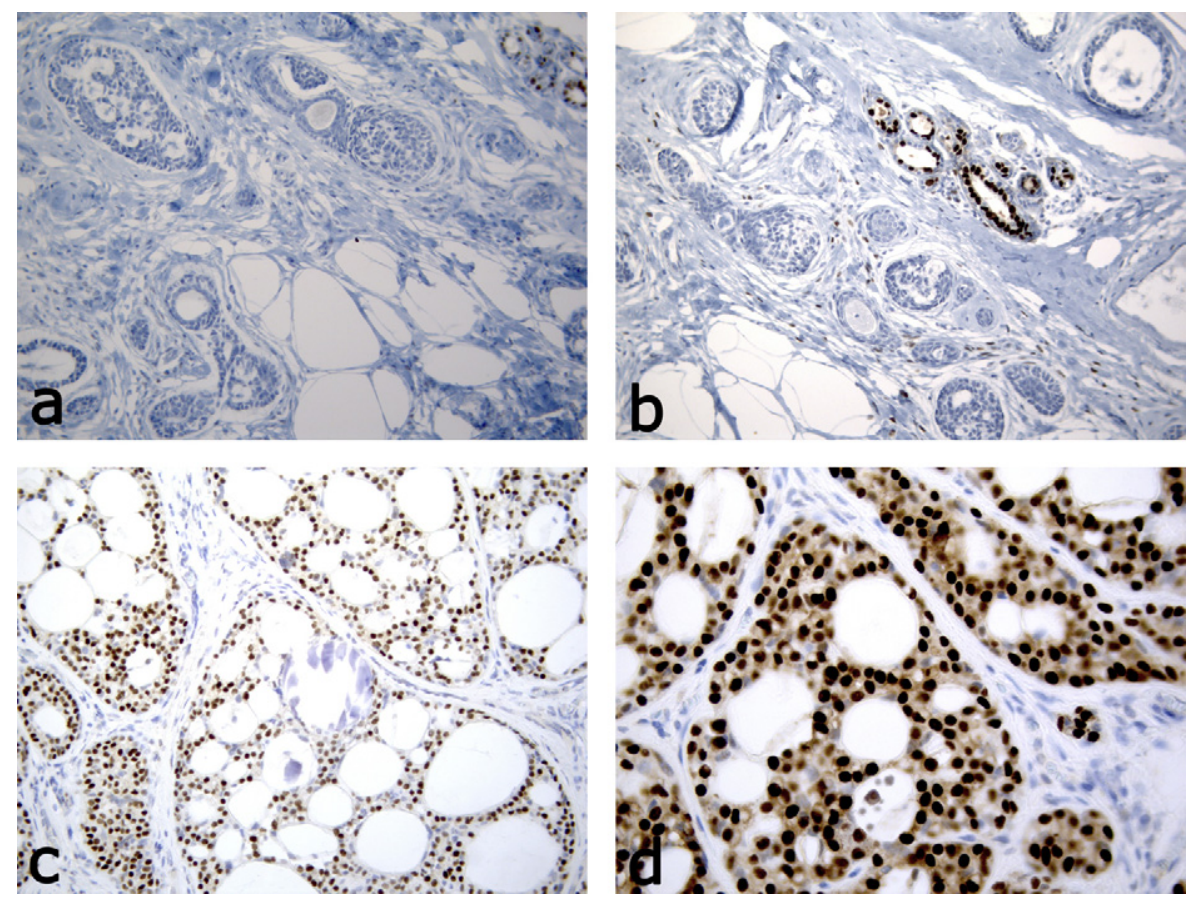

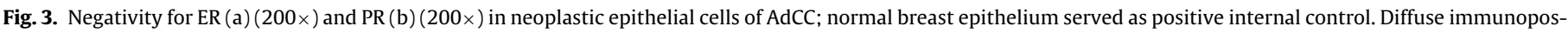
itivity for ER (c) $(200 \times)$ and PR (d) $(400 \times)$ in epithelial cells of CS.

CS, CD10, HHF35, PR and ER were negative in all the AdCC cases (Figs. 3 and 4).

\section{Discussion}

A cribriform growth pattern is commonly found in a heterogeneous variety of breast lesions, such as invasive cribriform carcinoma, cribriform ductal in situ carcinoma, adenoid cystic carcinoma and Collagenous Spherulosis. The differential diagnosis between AdCC and CS is important, due to the necessity of a surgical therapy in the case of AdCC, while no treatment is needed in CS. Some morphological features consent to distinguish these lesions on hematoxylin-eosin slides but, especially in bioptic specimens, the diagnosis can be challenging. In this setting, several studies have suggested the need for an immunohistochemical approach [11].

In AdCC two cell types are present: myoepithelial-like cells, which represent the majority and are responsible for the cribriform growth pattern, and epithelial cells, forming ductules with a random distribution. Cytologically, ductular epithelial cells 

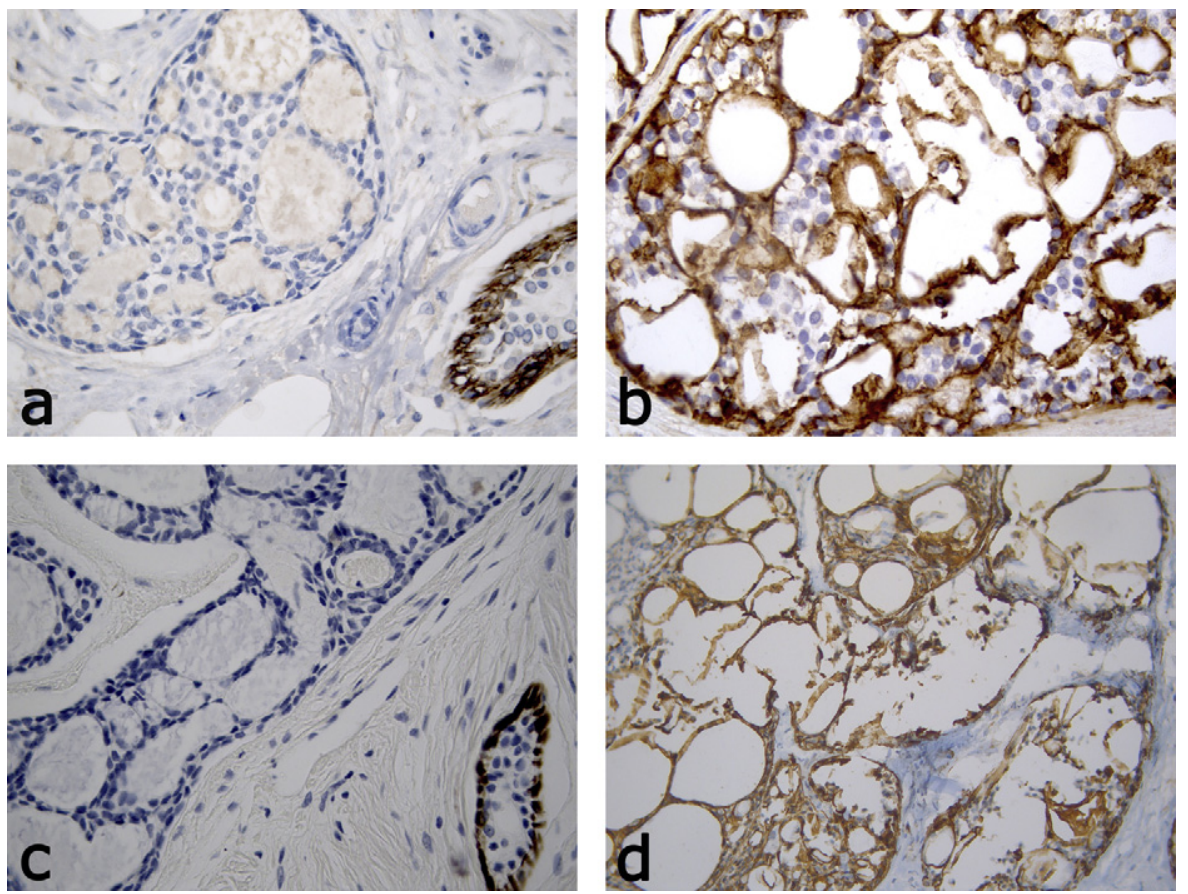

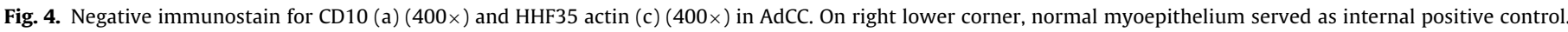
Diffuse immunopositivity for CD10 (b) $(400 \times)$ and HHF35 actin (d) $(200 \times)$ in the inner myoepithelial flat layer and in the cuticle amorphous material of CS.

are characterized by eosinophilic cytoplasm, while the more abundant myoepithelial-like cells have a weakly basophilic cytoplasm. Ductular epithelial structures are sometimes scanty, with a small diameter, and mainly when the specimen is inconspicuous, they are difficult to recognize. This could lead to a potential misdiagnosis of AdCC as cribriform invasive carcinoma or cribriform ductal in situ carcinoma [11]. Moreover, when the pseudoglandular spaces are particularly represented and contain abundant basement membrane material, the AdCC could be misdiagnosed as CS.

In $\mathrm{CS}$, the spaces are bordered by a peripheral eosinophilic cuticle of variable thickness and staining intensity and are surrounded by a row of myoepithelial cells. Diagnosis could be very difficult when the eosinophilic cuticle is not evident or when CS coexists with other entities, such as ductal hyperplasia or lobular in situ carcinoma. So the immunohistochemical assessments become essential.

Unfortunately, in the differential diagnosis between AdCC and $\mathrm{CS}$, an immunophenotypic overlap of SMA, p63 and CK 5/6 has been reported $[1,3,11,12]$. Our findings were in keeping with these data.

Recently, to better differentiate between the two lesions, Rabban et al. proposed the evaluation of calponin and smooth muscle myosin, both positive in CS and negative in AdCC [11].

Our study suggests that two other myoepithelial markers, CD10 and HHF-35, strongly differ in the two lesions, revealing to be useful in the differential diagnosis. In fact, they were expressed in myoepithelial cells of CS but were absent in AdCC. To our knowledge, CD10 and HHF-35 actin expression has been investigated in salivary glands AdCC [10], but no studies are reported on breast AdCC.

Moreover, our immunohistochemical findings, in addition to data reported in the literature $[1,5,7,11,13]$, lead to hypothesize that AdCC could be a true basal-like neoplasia, even if with a more favorable prognosis, composed by two different cell types, probably arising from a basal stem line tending to divergent and incomplete differentiation toward epithelial basal-like CK5/6/Ckit+, ER/PR-cell type and toward myoepitelial-like cell type, with an incomplete SMA/p63+, CD10/HHF35-immunophenotype. The latter could lose CD10 and HHF35 expression during the multistep process of oncogenesis.

By contrast, CS represents a reactive, benign, proliferation of two well-differentiated cell types: myoepithelial, p63/SMA/ CD10/HHF35 positive, cells, (as in normal myoepithelium of the breast) and epithelial, C-kit-, ER/PR+, cells [2,12].

In conclusion, our results give evidence of the usefulness of a broad immunohistochemical panel, including CD10 and HHF35 actin, to distinguish AdCC and CS, which due to their different prognosis need a different treatment. A complete immunophenotypic panel can also be helpful to investigate the histogenesis of the two lesions.

\section{References}

[1] S. Azoulay, M. Lae, P. Freneaux, et al., KIT is highly expressed in adenoid cystic carcinoma of the breast, a basal-like carcinoma associated with a favourable outcome, Mod. Pathol. 18 (2005) 1623-1631.

[2] P.B. Clement, R.H. Young, J.G. Azzopardi, Collagenous Spherulosis of the breast, Am. J. Surg. Pathol. 11 (1987) 411-417.

[3] D.X.G. Divaris, S. Smith, D. Leask, et al., Complex Collagenous Spherulosis of the breast presenting as a palpable mass, Breast J. 6 (2000) 199-203.

[4] B.A. Gusterson, P. Monaghan, R. Mahendran, J. Ellis, M.J. O'Hare, Identification of myoepithelial cells in human and rat breasts by anti-common acute lymphoblastic leukemia antigen antibody A12, J. Natl. Cancer Inst. 77 (1986) 343-349.

[5] B. Haupt, J.Y. Ro, M.R. Schwartz, Basal-like breast carcinoma: a phenotypically distinct entity, Arch. Pathol. Lab. Med. 134 (2010) 130-133.

[6] S.A. Hoda, P.P. Rosen, Observations on the pathologic diagnosis of selected unusual lesions in needle core biopsies of breast, Breast J. 10 (November-December (6)) (2004) 522-527.

[7] M.G. Mastropasqua, E. Maiorano, G. Pruneri, et al., Immunoreactivity for c-kit and $\mathrm{p} 63$ as an adjunct in the diagnosis of adenoid cystic carcinoma of the breast, Mod. Pathol. 18 (2005) 1277-1282.

[8] E.E. Mooney, N. Kayani, F.A. Tavassoli, Spherulosis of the breast. A spectrum of mucinous and collagenous lesions, Arch. Pathol. Lab. Med. 123 (1999) 626-630.

[9] S. Moritani, R. Kushima, H. Sugihara, M. Bamba, T.K. Kobayashi, T. Hattori, Availability of CD10 immunohistochemistry as a marker of breast myoepithelial cells on paraffin sections, Mod. Pathol. 15 (2002) 397-405.

[10] O. Neves Cde, A.B. Soares, A.F. Costa, V.C. de Araujo, C. Furuse, P.B. Juliano, A. Altemani, CD10 (neutral endopeptidase) expression in myoepithelial cells 
of salivary neoplasms, Appl. Immunohistochem. Mol. Morphol. 18 (2010) $172-178$.

[11] J.T. Rabban, R.S. Swain, C.J. Zaloudek, D.R. Chase, Y.Y. Chen, Immunophenotypic overlap between adenoid cystic carcinoma and Collagenous Spherulosis of the breast: potential diagnostic pitfalls using myoepithelial markers, Mod. Pathol. 19 (2006) 1351-1357.
[12] E. Resetkova, C. Albarracin, N. Sneige, Collagenous Spherulosis of breast: morphologic study of 59 cases and review of the literature, Am. J. Surg. Pathol. 30 (2006) 20-27.

[13] Y. Yamamoto, M. Ibusuki, M. Nakano, T. Kawasoe, R. Hiki, H. Iwase, Clinical significance of basal-like subtype in triple-negative breast cancer, Breast Cancer 16 (2009) 260-267. 